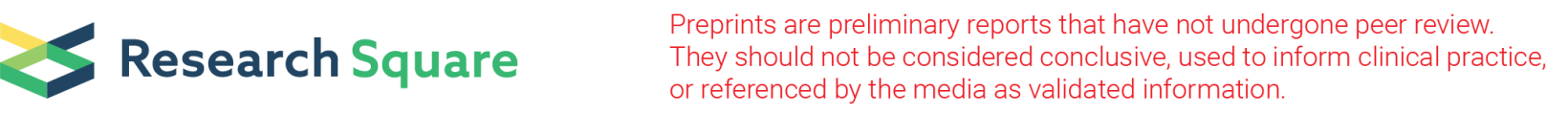

\title{
Factors Associated With Adherence of Iron Folic Acid Supplementation Among Pregnant Women in Rural Soro District, Hadiya Zone, Ethiopia: Cross Sectional Study
}

\section{Tegegn Tadesse Arficho ( $\square$ tegegntadesse24@gmail.com ) \\ Wachamo University}

\section{Research Article}

Keywords: Adherence, Iron, Folic acid, pregnant women, Ethiopia

Posted Date: March 2nd, 2022

DOI: https://doi.org/10.21203/rs.3.rs-1350549/v1

License: (9) This work is licensed under a Creative Commons Attribution 4.0 International License.

Read Full License 


\section{Abstract}

Background: Iron deficiency anemia is a wide spread public health problem around the globe which accounts $20 \%$ of maternal death worldwide. World health organization recommends every pregnant woman to take a daily dose of $30-60 \mathrm{mg}$ of iron and $400 \mu \mathrm{g}$ of folic acid tablets starting from early months of pregnancy. Despite the advantages of iron and folic acid supplementation the proportion of pregnant women who took iron supplements for 90 days or more during their last pregnancy was very low. Therefore this study assessed the adherence to iron and folic acid supplementation and its associated factors among pregnant women in rural Soro district, Hadiya Zone, Southern Ethiopia.

Methods: Cross sectional study was employed to assess the compliance and factors associated with compliance of iron folic acid among women who gave birth one year prior to the date of survey. Simple random sampling method was applied to select the study subjects. Both bivariate and multivariable logistic regression was used to assess the association between the study variables and to control the possible confounding.

Results: From the whole study participants only 51(18.8\%) women had taken iron folic acid supplements for at least 90 days during their last pregnancy. On multivariable logistic regression analysis after adjusting for potential confounders women who had ANC of 4 and above times were 6.8 times more likely to take the minimum optimum dose of iron folic acid. Women who were frequently visiting health facilities for ante natal care were more likely to adhere for iron folic acid supplements than their counterparts.

Conclusion: In this study high proportion of pregnant women did not take adequate dose of iron folic acid tablets during their last pregnancy. Every effort should be done to mobilize pregnant women to take the minimum acceptable dose of IFA during their pregnancy. Additional channels to provide IFA supplements for pregnant women should be considered in addition to providing free IFA tablets ANC contact with mothers.

\section{Introduction}

Around $41.8 \%$ of pregnant women in the world are anemic. Iron deficiency is the primary cause of anemia in pregnant women and iron deficiency anemia accounts at least for about one half of the all cases of anemia globally. Physiological requirement for iron is highest during pregnancy and iron from diets taken does not fulfill an individual's requirement. Therefore it is important to routinely supplement all pregnant women with iron supplements. In middle and low income countries iron and folic acid supplementation is very important intervention among strategies formulated to prevent and reduce anemia during pregnancy $[1,2]$. World health organization (WHO) recommends every pregnant woman to take a daily dose of 30$60 \mathrm{mg}$ of iron and $400 \mu \mathrm{g}$ of folic acid tablets starting from early months of pregnancy as integral part of ANC program. It is ideal to ingest 180 iron folic acid tablets to prevent iron and folate deficiencies before 
delivery. Currently many countries are working to achieve pregnant women to take 90 and more tablets of IFA during pregnancy $[1,3,4,5]$.

Adequate intake of iron and folic acid has several benefits for both mothers and their children. Increasing coverage and adherence to IFA supplements are very important to get their impact. Similar to WHO recommendation Ethiopian national protocol on IFA supplementation recommends that all pregnant women should take $30-60 \mathrm{mg}$ of IFA tablets per day for six months starting as early as possible. It indicates that every pregnant mother should take IFA supplements at least for 90 days during pregnancy. It is given as integral part of ANC and provided free of charge [5-7].

Iron deficiency anemia is a wide spread public health problem around the globe which accounts $20 \%$ of maternal death worldwide. Anemia during pregnancy is highest in Africa with $61.3 \%$ of pregnant women are anemic especially in Sub Saharan Africa. Around 17.2 million people are affected by anemia, which corresponds to approximately $30 \%$ of total global cases [8]. Studies show that 22 to $56.8 \%$ of pregnant women are anemic in Ethiopia with higher proportion of women affected in rural areas $[4,6,9]$. It indicates that iron deficiency anemia is a moderate to severe public health problem in Ethiopia. Anemia, maternal and child mortality, prenatal and perinatal loss, low birth weight and prematurity occur due to iron deficiency in child bearing age $[1,5,8]$.

Government of Ethiopia developed nutrition strategies and programs to routinely provide iron and folic acid supplementation for pregnant women with the target of providing daily IFA tablet for six months (sometimes it could include 3months postpartum period). It is aimed at increasing proportion of pregnant women supplemented with IFA more than 50\% $[7,10]$. However, 2016 EDHS report indicated that only five percent of women got iron supplementation for at least 90 days during their pregnancy [4]. Ethiopia is one of the countries with lowest level of coverage and adherence of iron folic acid supplementation among pregnant women even though WHO recommends that every pregnant woman should receive supplements of iron and folic acid.

Several studies show that age of the mother, educational status of the mother, knowledge of anaemia and iron folate tablets, history of anaemia and household wealth index are identified factors associated with compliance to iron and folic acid supplements in Ethiopia [11, 12, 13]. Moreover in southern Ethiopia no study done on assessing compliance of IFA depending on national protocol which recommends that the tablets should be taken for at least 90 days during pregnancy. Therefore this study assessed the status of and factors associated with adherence of prenatal iron folic acid supplementation among women during pregnancy in rural Soro district, Hadiya Zone, South Ethiopia.

\section{Objectives}

\section{General objective}

To assess the status of adherence for iron folic acid supplementation and its associated factors among pregnant women in rural Soro district, Hadiya Zone, Ethiopia 2018 


\section{Specific objectives}

1. To determine the status of adherence for iron folic acid supplementation among pregnant women

2. To identify factors associated with iron folic acid supplementation among pregnant women

\section{Methods}

\section{Study setting}

Sorro district is one of the ten woradas and two urban adminstrations in Hadiya zone. It is located in $262 \mathrm{~km}$ from Addis Ababa and $32 \mathrm{~km}$ from zonal capital, Hossana. The total population of Sorro district is 268281 and female accounts $134673(50.2 \%)$ as reported by the district's finance and economic development office in 2017G.C. The total number of households at the district estimated to be 29126 [14].

\section{Study design}

Community based cross sectional study was employed to assess the status of and factors associated with compliance of iron folic acid supplements among pregnant women at the study area.

\section{Inclusion and Exclusion criteria}

All women who lived in rural Soro district at least for 6 months and gave live birth 12 months prior to the survey. Mothers who were severely sick and unable to respond excluded from the study. Additionally mothers who can't talk and hear due to disability were not included in the study.

\section{Source and study population}

The source population was all mothers who live in rural Soro district and gave birth one year prior to the date of interview. The study population of this study was all mothers who gave birth within one year prior to the date of survey and selected for the study.

\section{Sample Size determination}

To estimate the sample size for compliance of IFA supplementation among women single proportion formula was used by using the following assumptions: Anticipated Proportion of mothers compliant of IFA supplementation is $20.4 \%$ [12], $5 \%$ type I error, margin of error $5 \%$ and $10 \%$ contingency for the non response. By considering non-response rate of $10 \%$, the final sample size was 274 women who gave birth 12 months before the date of the survey.

\section{Sampling Techniques and Procedures}

Soro district was chosen purposively out of ten rural districts in Hadiya Zone. Then twelve rural kebeles (smallest administrative units) were selected randomly by using lottery method out of 46 rural kebeles found in Sorro district. The list of households having women with children less than one year was 
registered by using health development armies from each selected kebeles. Then the sample size was allocated proportionally for each kebele. After preparing the sampling frame simple random sampling method was applied to collect data from the index mother.

\section{Operational definition}

A mother was categorized as adherent for IFA supplements during pregnancy if she has reported as at least 90 doses of iron folic acid supplements were taken during her last pregnancy [15].

\section{Data collection procedures}

Quantitative data were collected by using pretested structured questionnaire adopted from EDHS and different literatures which were designed to assess maternal health care practices in developing countries. Questionnaire was prepared by English language and translated to local language, Hadiyissa and back translated to English by fluent speakers of the two languages. Intensive training was given for supervisors and data collectors on the purpose of study, how to handle questionnaires, how to conduct data collection and on ethical consideration. Then data were collected by using twelve trained female data collectors who were fluent in local language Hadiyissa with prior experience of participation in data collection. Four BSc health professionals were participated on supervision of the data collection process. Five percent of the questionnaires were pre tested before actual data collection period. Strict supervision was done by supervisors and the overall quality of the data collection was monitored by investigators of the study. Data were collected from June 10 up to 20, 2018. Data collectors submit daily collected data for supervisors and the collected data had been checked for completeness, consistency and any error was being corrected immediately.

\section{Variables}

Dependent variable: adherence of IFA tablets during pregnancy

Independent variables:

Socio demographic and economic characteristics, maternal health service related characteristics, obstetric characteristics and knowledge of IFA supplementation and anemia. Knowledge on anemia was assessed by asking questions about causes, symptoms, prevention and treatment of anemia. Similarly knowledge according to IFA was assessed by asking questions about uses of IFA tablets, duration of adequate intake, time of starting supplements and side effects. Correct answer scored one point and incorrect answer scored zero. Those who had correctly answered more than $80 \%$ of anemia and iron folic assessing questions were decided as having high knowledge on anemia and IFA. Additionally those mothers who had correctly responded $60-80 \%$ and less than $60 \%$ of knowledge assessing questions were also categorized as medium and low knowledge of anemia and IFA respectively [12].

\section{Data processing, analysis and presentation}


After field work data was checked for completeness and consistence before data entry and cleaning. Then Data was entered on Epi info version 3.5.4 and exported to SPSS for windows version 16 (SPSS Inc. version 16, Chicago, Illinois) to do analysis. Descriptive summary was presented by using frequencies, proportions, means and tables. Household socioeconomic data was collected based on household assets and analyzed by using principal component analysis. Both bivariate and multivariable logistic regression analysis was used to assess the association of independent variables with outcome variable and to control the possible confounding factors. Compliance of IFA supplements was coded as "1" while noncompliance of IFA supplements was coded as " 0 " for running logistic regression analysis. Odds ratio with $95 \%$ confidence interval was used to assess strength of association. Any significantly associated variable with $p$-value less than or equal to 0.25 during bivariate analysis was candidate for multivariable analysis. Finally during multivariable analysis an independent variable with P-value less than 0.05 was considered as statistically significant and an independent predictor of compliance of iron supplements during pregnancy.

\section{Result}

\section{Socio demographic characteristics of respondents}

From the total of 274 women who were allocated for the study 271 were participated at the survey making the response rate of $98.9 \%$. The mean age of women was $26.83( \pm 5.25)$ which ranges from 15 up to 43 years. More than half of the mothers 151 (55.7\%) were found within age of 25-34 years. According to the marital status most of the respondents $259(95.6 \%)$ were married and protestant religion followers $245(90.4 \%)$ contains most of the proportion. Similarly most of the respondents were Hadiya $245(90.4 \%)$ in their ethnicity and two third (75.3\%) were housewives in their occupation. Around half of their husbands (50.6\%) and fourthy one percent of the women had learnt primary education. Most of the mothers $189(69.7 \%)$ reported as they didn't know the average monthly income of the house hold. More than two third of the respondents $239(88.2 \%)$ answered as they have their own livestock, herds other farm animals or poultry (Table 1 ). 
Table 1

The Socio demographic characteristics of mothers in Sorro district, Hadiya Zone, South Ethiopia, 2018

\begin{tabular}{|c|c|c|c|}
\hline Variable & Category & No & percent \\
\hline \multirow[t]{3}{*}{ Age of mother } & $15-24$ & 91 & 33.6 \\
\hline & $25-34$ & 151 & 55.7 \\
\hline & $\geq 35$ & 29 & 10.7 \\
\hline \multirow[t]{2}{*}{ Religion of mother } & Protestant & 245 & 90.4 \\
\hline & Other $^{1}$ & 26 & 9.6 \\
\hline \multirow[t]{3}{*}{ Mother's ethnicity } & Hadiya & 245 & 90.4 \\
\hline & Kambata & 13 & 4.8 \\
\hline & Tambaro & 13 & 4.8 \\
\hline \multirow[t]{4}{*}{ Occupation of mother } & housewife & 204 & 75.3 \\
\hline & merchant & 44 & 16.2 \\
\hline & farmer & 11 & 4.1 \\
\hline & Other* & 12 & 4.4 \\
\hline \multirow{5}{*}{$\begin{array}{l}\text { Mother's educational } \\
\text { Status }\end{array}$} & Illiterate & 107 & 39.5 \\
\hline & Read and write only & 29 & 10.7 \\
\hline & primary & 113 & 41.7 \\
\hline & Secondary and above & 22 & 8.1 \\
\hline & Never heard & 148 & 54.6 \\
\hline \multirow[t]{5}{*}{ Wealth index } & poorest & 67 & 24.7 \\
\hline & poor & 58 & 21.4 \\
\hline & middle & 63 & 23.2 \\
\hline & rich & 47 & 17.3 \\
\hline & richest & 36 & 13.3 \\
\hline
\end{tabular}


Around two third of women 208(76.8\%) had taken at least one time of ANC visit during the last pregnancy. Eighty six (31.7\%) women were not given or bought any iron and folic acid supplements during their pregnancy. More than half of the women reported as consumed at least one tablet of IFA during their last pregnancy with median number of 45 supplements which ranges from 2 up to 140 tablets. The mean month of pregnancy to start the first tablet was at $5.98( \pm 1.82)$ month of pregnancy. From the whole study participants only $51(18.8 \%)$ of the women were compliant of iron and folic acid (IFA) supplementation during their last pregnancy. Majority of respondents $220(81.2 \%)$ had taken IFA supplements for less than 90 days or did not take any tablet during the whole pregnancy. From those who had given IFA during pregnancy more than half the mothers took 1-59 tablets.

Negligence (poor attention to importance of IFA)85(31.4\%), forgetfulness 36(13.3\%) and fear of side effects $25(9.2 \%)$ were the main reasons for not taking the given IFA supplements daily or to totally deny receiving IFA tablets during the last pregnancy. According to the knowledge of women $244(90 \%)$ and $167(61.6 \%)$ of women had high knowledge of anemia and iron folic acid supplementation respectively (Table 2). 
Table 2

Maternal health service and obstetric related characteristics in Sorro district, Hadiya Zone, South Ethiopia, 2018. ( $=271)$

\begin{tabular}{|c|c|c|c|}
\hline Variable & Category & No & Percent \\
\hline \multirow[t]{2}{*}{ Nearest health facility } & Health post & 181 & 66.8 \\
\hline & Health center & 90 & 33.2 \\
\hline \multirow[t]{3}{*}{ No of children currently } & 1 up to 3 children & 115 & 42.4 \\
\hline & 4 up to 6 children & 118 & 43.5 \\
\hline & 7 and above & 38 & 14.0 \\
\hline \multirow[t]{3}{*}{ Birth order } & first & 31 & 11.4 \\
\hline & second & 50 & 18.5 \\
\hline & Third and above & 190 & 70.1 \\
\hline \multirow[t]{2}{*}{ ANC received } & yes & 208 & 76.8 \\
\hline & no & 63 & 23.2 \\
\hline \multirow[t]{4}{*}{ No of ANC } & One time & 9 & 3.3 \\
\hline & $2-3$ times & 115 & 42.4 \\
\hline & 4 and above & 72 & 26.6 \\
\hline & I don't know & 12 & 4.4 \\
\hline \multirow[t]{2}{*}{ IFA given } & yes & 180 & 66.4 \\
\hline & no & 91 & 33.6 \\
\hline \multirow[t]{2}{*}{ Compliant of IFA } & yes & 220 & 81.2 \\
\hline & no & 51 & 18.8 \\
\hline \multirow[t]{3}{*}{ IFA by categories } & 1-59 tablets & 110 & 59.5 \\
\hline & $60-89$ tablets & 32 & 17.3 \\
\hline & 90 and above & 43 & 23.2 \\
\hline \multirow[t]{3}{*}{ Knowledge of anemia } & Low knowledge & 9 & 3.3 \\
\hline & Medium knowledge & 15 & 6.6 \\
\hline & High knowledge & 244 & 90.0 \\
\hline \multirow[t]{2}{*}{ Knowledge of IFA } & Low knowledge & 86 & 31.7 \\
\hline & Medium knowledge & 18 & 6.6 \\
\hline
\end{tabular}




\begin{tabular}{|llll|}
\hline Variable & Category & No & Percent \\
\hline High knowledge & 167 & 61.6 \\
\hline
\end{tabular}

\section{Factors associated with optimal iron folic acid supplementation}

Bivariate analysis revealed that mother's educational level being secondary and above $[\mathrm{COR}(95 \% \mathrm{Cl})=$ $2.19(1.08,4.43)]$, husband's educational status being secondary and above $[\mathrm{COR}(95 \% \mathrm{Cl})=$ $3.09(1.15,8.27)]$ and having ANC of four and above times $[\mathrm{COR}(95 \% \mathrm{Cl})=7.17(2.01,25.6)]$ were factors significantly associated with compliance of IFA supplementation. On multivariable logistic regression analysis after adjusting for potential confounders having ANC of 4 and above times was significantly associated with optimal iron folic acid supplementation (at least 90 days) in pervious last recent pregnancy among women. Those pregnant women who had four and above visit for ANC were 6.8 times more likely to take iron folic acid supplements for at least 90 days than those women who did not attend antenatal care $[\mathrm{AOR}(95 \% \mathrm{Cl})=6.80(1.85,25.04)]($ Table 3$)$. 
Table 3

Factors associated with adherence of IFA among pregnant women in Sorro district, Hadiya Zone, South Ethiopia, 2018

\begin{tabular}{|c|c|c|c|c|}
\hline \multirow[t]{2}{*}{ Variable } & \multicolumn{2}{|c|}{ Compliant of IFA } & \multirow[t]{2}{*}{$\operatorname{COR}(95 \% \mathrm{Cl})$} & \multirow[t]{2}{*}{$\mathrm{AOR}(95 \% \mathrm{Cl})$} \\
\hline & yes & no & & \\
\hline \multicolumn{5}{|l|}{ Maternal education } \\
\hline Illiterate & $4(13.8)$ & $93(86.9)$ & 1 & 1 \\
\hline Write and read only & $14(13.1)$ & $25(86.2)$ & $1.06(0.32,3.51)$ & $0.78(0.22,2.82)$ \\
\hline Primary & $28(24.8)$ & $85(75.2)$ & $2.19(1.08,4.43)^{\star}$ & $1.36(0.61,3.03)$ \\
\hline $\begin{array}{l}\text { Secondary and } \\
\text { above }\end{array}$ & $5(22.7)$ & 17(77.3) & $1.95(0.62,6.14)$ & $0.78(0.19,3.11)$ \\
\hline \multicolumn{5}{|l|}{ Husbands education } \\
\hline Illiterate & $7(12.3)$ & $50(87.7)$ & 1 & 1 \\
\hline Write and read only & $6(25)$ & $18(75.0)$ & $2.38(0.71,8.03)$ & $2.26(0.61,8.37)$ \\
\hline Primary & $22(16.1)$ & 115(83.9) & $1.37(0.55,3.40)$ & $1.05(0.40,2.77)$ \\
\hline Secondaryand above & $16(30.2)$ & $37(69.8)$ & $3.08(1.15,8.27)^{\star}$ & $2.71(0.85,8.63)$ \\
\hline \multicolumn{5}{|l|}{ Antenatal care } \\
\hline No ANC & $3(4.8)$ & $60(95.2)$ & 1 & 1 \\
\hline $1-3$ times & $27(21.8)$ & $97(78.2)$ & $5.57(1.62,19.15)$ & $5.43(1.52,19.44)$ \\
\hline 4 and above ANC & $19(26.4)$ & $53(73.6)$ & $7.17(2.01,25.60)^{\star \star}$ & $6.74(1.83,24.82)^{\star \star}$ \\
\hline I don't know & $2(16.7)$ & $10(83.3)$ & $4(0.60,27.02)$ & $4.15(0.60,29.15)$ \\
\hline
\end{tabular}

\section{Discussion}

This study was concerned with assessing the compliance and factors associated with compliance of iron folic acid among pregnant women. The status of compliance of IFA was $18.8 \%(95 \% \mathrm{Cl}: 14.8,22.8)$ in this study. The finding is similar with the previous study findings in Pakistan $16.9 \%$, Kenya $(18.3 \%)$ and Northern Ethiopia $(20.4 \%)[12,16,17]$. However the finding of this study is higher than the prevalence of compliance of IFA reported in EDHS 2011 which was only 5\% [4]. In contrary to this, the current study finding is lower than those indicated in studies conducted in Senegal (51\%), Nepal (23\%), India (23.8\%) and Indonesia $(34 \%)[15,18,19]$. This difference might be due to methodological and socio cultural differences among study subjects. 
In multivariable logistic regression it is showed that number of ANC was found to be a factor associated with consuming minimum dose of iron folic acid among pregnant women. Those pregnant mothers who had four and more times of visit for antenatal care were more likely to be compliant of IFA than those had no ANC follow up. Iron and folic acid is given as the integral part of ANC with free of charge in Ethiopia. The increased odds of using IFA supplements among mothers with at least minimum recommended number of ANC might be due to the increased chance of getting supplements and counseling from the health workers during contact at appointments. It is also the only channel for providing free iron folic acid supplements for pregnant women in Ethiopia. Lack of access to get the IFA supplements through optional ways like home to home distribution might be another reason among those mothers with no ANC follow up to be none compliant of supplementation than their counterparts. It is in line with findings from studies done in India and Kenya $[16,17]$ that indicated ANC visits $\geq 4$ times is an important predictor of optimum supplementation.

This study has its own limitations. The study was conducted by self-reporting which overestimates compliance as compared with pill count. The result of this study could be prone to recall bias because of error in recalling the past history related to their last pregnancy. However every effort was made to reduce the occurrence of the bias. Another weakness of this study is using of small sample size to undertake the study.

\section{Conclusion}

In this study high proportion of pregnant women did not take adequate dose of iron folic acid tablets during their last pregnancy. Every effort should be done to mobilize pregnant women to take the minimum acceptable dose of IFA during their pregnancy. Additional channels to provide IFA supplements for pregnant women should be considered in addition to providing free IFA tablets ANC contact with mothers.

\section{List Of Abbreviations}

AOR Adjusted odds ratio

ANC Antenatal care

COR Crude odds ratio

EDHS Ethiopian demographic health survey

IFA Iron folic acid

SPSS Statistical package for social sciences

WHO World health organization 


\section{Declarations}

\section{Ethical approval and consent to participate}

Ethical clearance was obtained from the Wachemo University, college of medicine and health sciences ethical committee. The letter of cooperation was written for Sorro District health office in order to proceed the study. The study was conducted based on voluntary participation by study subjects after explaining the purpose of study. Informed consent was obtained from each participant before starting the interview without any obligation or persuading. Anyone had right to withdraw from interview at any time without any harm. No name of participant has been written and code was used instead and confidentiality of data was assured for participants. Privacy and confidentiality of personal information of research subjects have been ensured during the study. The overall method was preformed according to the world medical association (WMA) regulations and principles on research involving human participants.

\section{Acknowledgment}

I would like to thank data collectors, supervisors, respondents and all individuals involved for their contribution.

\section{Availability of data and material}

The datasets are available from the corresponding author on reasonable request.

\section{Authors' contribution}

All components of the research were done by TT

\section{Financial disclosure}

There was no funding source.

\section{Competing interest}

The author declares that there is no competing interest.

\section{Consent for publication}

Not applicable.

\section{References}

1. WHO Guideline. Daily iron and folic acid supplementation in pregnant women. Geneva, World Health Organization. 2012.

2. SPRING nutrition technical brief. A rapid initial assessment of the distribution and consumption of iron -folic acid tablets through antenatal care in Ethiopia, September. 2014. 
3. SPRING nutrition technical brief: A Rapid Initial Assessment of the Distribution and Consumption of Iron-Folic Acid Tablets through Antenatal Care in Benin. Arlington, VA: USAID/Strengthening Partnerships, Results and Innovations in Nutrition Globally (SPRING) Project.

4. Central Statistical Agency [CSO, Ethiopia] and ICF International. Ethiopia Demographic and Health Survey 2016. Addis Ababa, Ethiopia and Calverton, Maryland, USA: Central Statistical Agency and ICF International.

5. Federal Ministry of Health of Ethiopia (FMOH). National Guideline for Control and Prevention of Micronutrient Deficiencies, FMOH, Addis Ababa, Ethiopia, 2004.

6. Gebremedhin S, Samuel A, Mamo G, Moges T, Assefa Ts: Coverage compliance and factors associated with utilization of iron supplementation during pregnancy in eight rural districts of Ethiopia. BMC Public Health. 2014; 14: 607.

7. FMOH. National nutrition strategy. Addis Ababa, Ethiopia. January 2008.

8. Bekele A, Tilahun M, Mekuria A. Prevalence of Anemia and Its Associated Factors among Pregnant Women Attending Antenatal Care in Health Institutions of Arba Minch Town, GamoGofa Zone, Ethiopia: A Cross-Sectional Study. 2016

9. Antony A. In utero physiology: role of folic acid in nutrient delivery and fetal development. Am J ClinNutr. 2007; 85:598-603.

10. Ogundipe O, Hoyo C, Ostbye B, Oneko O, ManongiR, Lie T, Daltveit K. Factors associated with prenatal folic acid and iron supplementation among 21,889 pregnant women in Northern Tanzania: A crosssectional hospital-based study. BMC Public Health. 2012; 12:481.

11. Taye B, Abeja G, Mekonen A. Factors associated with compliance of prenatal iron folate supplementation among women in Mecha district, Western Amhara: a cross-sectional study. Pan African Medical Journal. 2015; 20(43).

12. Haile D,Tabar L, LakewY. Differences in spatial distributions of iron supplementation use among pregnant women and associated factors in Ethiopia: evidence from the 2011 national population based survey. BMC pregnancy childbirth. 2017; 17(33).

13. Yasir Bin Nisar,Michael J Dibley,Ali Mohammad Mir. Factors associated with non-use of antenatal iron and folic acid supplements among Pakistani women: a cross sectional household survey. BMC Pregnancy and Childbirth. 2014; 14(305).

14. Soro District Finance and Economic Office report, Ethiopia. 2017.

15. Nisar B, Dibly J, Aguayo M. Iron-Folic Acid Supplementation During Pregnancy Reduces the Risk of Stunting in Children Less Than 2 Years of Age: A Retrospective Cohort Study from Nepal. Nutrients. $2016 ; 8(2): 67$.

16. Juma M, Shadrack O, Silvernus O. Predictors of optimum antenatal iron-folate supplementation in a low resource rural set-up in Eastern Kenya. Journal of Public Health and Epidemiology. 2015; 7(11):337-345.

17. Wendt A, Stephenson R, Young M, Webb-Girard A, Hogue C, Ramakrishnan U,Martorell R. Individual and Facility-Level Determinants of Iron and Folic Acid Receipt and Adequate Consumption among 
Pregnant Women in Rural Bihar, India. Plos One. 2015; 10(3).

18. Rialine T, John D. Factors associated with not using antenatal iron/folic acid supplements in Indonesia: the 2002/2003 and 2007 Indonesia Demographic and Health Survey. Asia Pac J ClinNutr 2015; 24(1):162-176

19. Khadim N, Adama F, Diégane T, Bintou D, Banda N, Bineta N, Papa N, Anta T. Determinants of Iron Consumption among Pregnant Women in Southern Senegal. Open Journal of Obstetrics and Gynecology. 201 7; 7:41-50. 